\title{
Radiologic Abnormalities and Autonomic Neuropathology in the Digestive Tract of the Ketonuric Diabetic Chinese Hamster
}

\author{
A. R. Diani ${ }^{1}$, D. M. Grogan ${ }^{1}$, M. E. Yates $^{1}$, D. L. Risinger ${ }^{2}$, and G. C. Gerritsen ${ }^{3}$ \\ ${ }^{1}$ Department of Anatomy, East Carolina University School of Medicine, Greenville, North Carolina, \\ ${ }^{2}$ Department of Radiology, Hillcrest Baptist Hospital, Waco, Texas, \\ and ${ }^{3}$ Diabetes and Atherosclerosis Research, The Upjohn Company, Kalamazoo, Michigan, USA
}

Summary. Barium x-ray patterns of ketonuric diabctic Chinese hamsters displayed marked dilatation of the stomach, small and large intestine. Hypomotility was manifested by flocculation of barium in the small and large bowel. Impaired transit time was further characterized by prolonged emptying of the stomach (mean $570 \mathrm{~min}$ diabetics; $200 \mathrm{~min}$ controls) and delayed stool formation (mean $230 \mathrm{~min}$ diabetics; and $100 \mathrm{~min}$ controls) and passage (mean $457 \mathrm{~min}$ diabetics; 210 min controls). Ultrastructural analysis of Auerbach's myenteric plexuses of the small intestine indicated acute degeneration in certain distal, unmyelinated axons. Swelling, deposition of glycogen, aggregation of neurofilaments and dense accumulation of lamellar bodies were observed. The severity and frequency of barium flocculation, glycogen deposition, aggregation of neurofilaments and lamellar inclusion bodies in axons were directly related to duration of ketonuria. The data strongly suggest that autonomic neuropathology in the plexuses of Auerbach may be a critical factor underlying gastrointestinal dysfunction in the ketonuric diabetic Chinese hamster.

Key words: Barium, ketonuric diabetic, Chinese hamster, dilatation, gastrointestinal, hypomotility, myenteric plexuses, autonomic neuropathology, diabetic neuropathy.

Several previous studies have shown abnormal radiologic patterns in the gastrointestinal tract of diabetic man. Oesophageal motor dysfunction [1], gastric dilatation and retention $[2,3,4,5]$ and impaired intestinal motility $[4,5,6,7]$ have been commonly reported. These complications may be accompanied by clinical symptoms such as diarrhoea or steatorrhoea $[4,6,7,8,9]$. It has been proposed by several investigators that the above anomalies were manifestations of autonomic neuropathy $[2,4$,
$6,7,8,9]$. Surprisingly, limited histological data have been reported to substantiate this relationship [10, $11,12,13,14,15]$. Ultrastructural data associated with gastrointestinal neuropathology are absent from the literature.

Recent light microscopic studies of the small intestine of nonketonuric and ketonuric diabetic Chinese hamsters demonstrated atony and distention, reduction in the number of Auerbach's plexuses [16] and decreased cholinesterase activity at neuromuscular junctions [17]. It has also been reported that diabetic Chinese hamsters displayed diarrhoea and steatorrhoea [18]. On the basis of these observations, barium $x$-ray analysis of the gastrointestinal tract of the ketonuric diabetic Chinese hamster was conducted to document any alterations in motility or gross radiologic abnormalities. Furthermore, the myenteric nerve plexuses in the small intestine were examined ultrastructurally to identify possible structural aberrations in the distal, autonomic axons.

\section{Materials and Methods}

Animals for this investigation were selected from the Upjohn colony of Chinese hamsters. Six pairs were designated for barium $\mathrm{x}$ ray analysis and 10 pairs for ultrastructural studies. Each pair consisted of a ketonuric diabetic matched with a nondiabetic control [18]. Diabetics were obtained from 7 different inbred sublines. Controls were derived from 3 sublines. Each pair was matched for body weight, sex and age. Mean \pm SEM body weight of the diabetics was $32 \pm 1 \mathrm{lg}$ (range 21-40) and the mean for controls was 32 $\pm \lg$ (range 25-39). Age varied from 8 to 18 months and duration of ketonuria from 2 to 9 months. All diabetic hamsters displayed continuous glycosuria as measured by consistent Tes-Tape ${ }^{\circledR}$ values of +3 to +4 . Ketonuria was determined by the Ketostix ${ }^{\bar{B}}$ method which rendered small, moderate and large values. All animals were provided Purina Mouse Breeder Chow and water ad libitum. The 6 pairs for radiologic experimentation were fasted for 4 hours prior to barium administration whereas the 10 pairs for ultrastructural studies were allowed food and water until sacrifice. Blood samples were drawn from the orbital sinus and analyzed for glucose by the Ames Eyetone ${ }^{\sqrt{3}}$. None of the diabetic animals received insulin 
Table 1. Radiologic time measurements

\begin{tabular}{|c|c|c|c|c|c|}
\hline \multicolumn{2}{|c|}{$\begin{array}{l}\text { Total emptying of gastric } \\
\text { barium (min) }\end{array}$} & \multicolumn{2}{|c|}{$\begin{array}{l}\text { Initial barium stool } \\
\text { formation ( } \mathrm{min})\end{array}$} & \multicolumn{2}{|c|}{$\begin{array}{l}\text { Initial barium stool } \\
\text { passage (min) }\end{array}$} \\
\hline Diabetic $^{a}$ & Control & Diabetic $^{a}$ & Control & Diabetic $^{a}$ & Control \\
\hline 630 & 270 & 210 & 90 & 300 & 140 \\
\hline 630 & 210 & 150 & 90 & 510 & 270 \\
\hline 630 & 150 & 270 & 90 & 500 & 90 \\
\hline 510 & 150 & 270 & 90 & 500 & 340 \\
\hline 510 & 150 & 270 & 150 & 420 & 250 \\
\hline 330 & 270 & 210 & 90 & 510 & 170 \\
\hline
\end{tabular}

${ }^{a} \mathrm{p}<0.01$ Diabetic vs. control

therapy at any time. The faecal contents were monitored for diarrhoea.

A Micropaque Powder ${ }^{\text {s }}$ aqueous solution of barium sulphate $(1.5 \mathrm{ml}$, specific gravity 1.3 ) was tube-fed directly into the stomach of each animal via a blunt 20 gauge needle attached to a $3 \mathrm{ml}$ syringe. Each animal was then restrained (prone position) in a transparent plastic tube $(14.7 \mathrm{~cm}$ long, $3.2 \mathrm{~cm}$ internal diameter). One end of the tube was closed with a fixed partition. Slots were located at $6.5 \mathrm{~cm}$ and $8.9 \mathrm{~cm}$ from the fixed end to accommodate a portable partition which compensated for variation in animal length. When each animal was correctly restrained, the plastic tube (with animal) was positioned on an $x$-ray table, $15.7 \mathrm{~cm}$ from the $\mathrm{X}$-ray source. A Picker GX600 x-ray apparatus was utilized with small focal spot and settings of 55 kilovolts and 400 milliamps. Xray exposures were made at 0.008 seconds to eliminate movement artifact. X-rays were recorded on Kodak XRP film which was housed in Kodak cassette C17 (regular intensifying screen). The film cassette was located directly beneath the restraining tube. All films were developed in a Kodak RPX-omat 90 second automatic processor. Each animal was x-rayed immediately after barium administration and at $30,90,150,210,270,330,510,630,1200$ and 1440 minutes. During the radiologic procedure, gastric emptying, initial barium stool formation in the colon and passage were observed and the time tecorded. X-rays of diabetic hamsters were analyzed for gross abnormalities and compared with controls by a radiologist who had no prior knowledge of animal background. The Fisher $t$ Test for Paired Means was used to analyze measurement data.

Animals for electron microscopy were sacrificed by exsanguination and decapitation. The small bowel of each animal was immediately excised, and divided into proximal, middle and distal thirds [16]. Tissue samples (approximately $10 \mathrm{~cm}$ long) were removed from the cranial region of the proximal and middle third. The tissues were then sliced into $1 \mathrm{~mm}$ blocks and immersed into $8 \%$ glutaraldehyde (Millonig's buffer, $\mathrm{pH} 7.4$ ) for 3 hours. After glutaraldehyde fixation, the intestinal blocks were rinsed in Millonig's buffer, post-fixed in $2 \%$ osmium tetroxide for $1 \frac{1 / 2}{2}$ hours, dehydrated and embedded in Epon. Several thick sections (approximately 1 micron) were taken from each block and stained with Paragon ${ }^{3}$ for light microscopic examination. If thick sections displayed myenteric nerve plexuses, serial thin sections (60 to 90 millimicrons) were cut, stained with uranyl acetate and lead citrate and examined in a Philips 201 electron microscope. Electron micrographs of Auerbach's plexuses in control and diabetic intestines were analyzed qualitatively and compared.

\section{Results}

Nonfasting blood sugars of 8 pairs were randomly obtained. Mean blood sugar of the diabetics was 371 $\pm 24 \mathrm{mg} / \mathrm{dl}$ (range 219-400) and the mean for nondiabetics was $113 \pm 12 \mathrm{mg} / \mathrm{dl}$ (range 75-170).
All diabetic animals showed intermittent to continuous diarrhoea characterized by loose, watery stools. Increased frequency and severity of diarrhoea (lack of stool formation) were observed in animals which had ketonuria for more than 3 months.

\section{Gross Pathology}

Post-mortem examination of the gastrointestinal tract of all diabetic animals showed distention and atony of the stomach and bowels compared with the alimentary canal of controls.

\section{Radiologic Abnormalities and Measurements}

Radiologic time measurements are presented in Table 1. Retention of material (Fig. 1 a), a combination of mucus and food, was observed in the stomach of $67 \%$ of the diabetics but was not observed in controls (Fig. 2a). Gastric emptying of barium was delayed in all diabetics (Table 1). Gastric dilatation was evident in all diabetics.

Reduced peristaltic activity and dilatation were evident in all diabetic bowels. The delay in transit was characterized by the flocculent appearance of barium (Fig. 1g), compared with controls (Fig. 2 g). Barium flocculation was most marked in animals with longterm ketonuria (7-9 months).

Initial barium stool formation in the colon was significantly retarded in all diabetics (Table 1). In addition, the diabetic stools were elongated and loose in texture compared with the short, compact stools of controls (Fig. 1i, 2i). Initial barium stool passage was significantly delayed in all diabetics (Table 1). Numerous stools and barium residue tended to aggregate in the diabetic colon as shown in the 1200 and $1440 \mathrm{~min}$ radiographs (Figs. $1 \mathrm{j}, 1 \mathrm{k}$ ). The colon of controls was generally devoid of barium stools by $1200 \mathrm{~min}$ (Fig. 2j). Clumping of stools was not observed in controls (Figs. $2 \mathrm{j}, 2 \mathrm{k}$ ). During the initial defaecation period (140 to $510 \mathrm{~min}$ ), the controls voided a mean of $10 \pm 3$ (range 2-16) stools compared with a significantly reduced mean of $3 \pm 0.5$ (range 1-4) for diabetics. During the later stages of 


\section{Ketonuric}

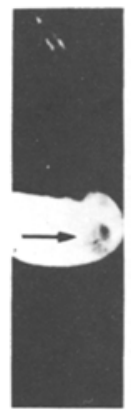

a

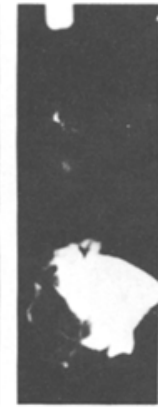

b

\section{Control}

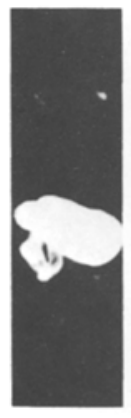

a

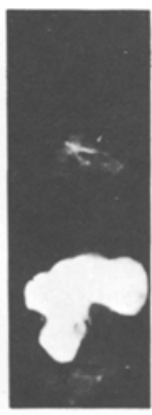

b

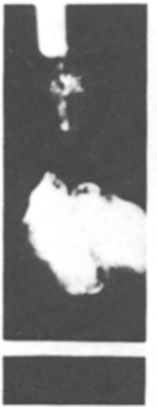

C

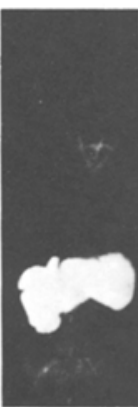

C

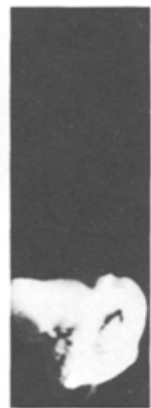

d

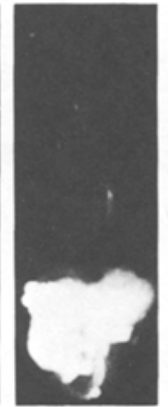

e

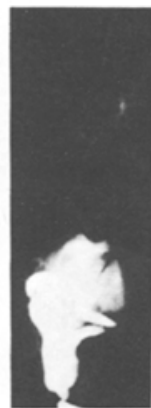

f

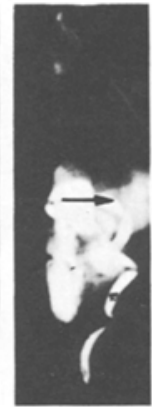

g

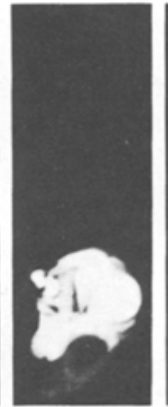

h

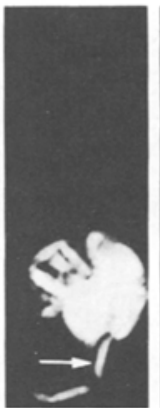

i

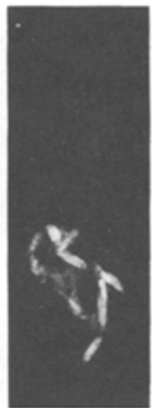

j

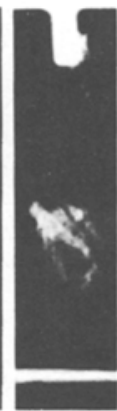

k

Figs. 1 and 2. Barium x-ray series of ketonuric and control hamsters. Letters a to $\mathrm{k}$ correspond to $0,30,90,150,210,270,330,510,630$, 1200 and 1440 minutes. Note the overall dilatation of the digestive tract of the ketonuric compared with the control (Figs. 1 a-, 2 a- $i$ ). Retention of mucus or food in the diabetic stomach is shown in Figure 1a (arrow). Figure $1 \mathrm{~g}$ (arrow) displays flocculation of barium in the ketonuric animal. Figure $2 \mathrm{~g}$ (arrow) indicates compact barium in the control. Note the elongated, loose stools of the ketonuric in Figure $1 \mathrm{i}$ (arrow) and the short, compact stools of the control in Figure $2 \mathrm{i}$ (arrow). Note the clumping of barium stools and barium residue in the ketonuric colon (Figs. $1 \mathrm{j}, 1 \mathrm{k}$ ) compared with the relatively clear colon in the control (Figs. $2 \mathrm{j}, 2 \mathrm{k}$ )

defaecation (510 to $1440 \mathrm{~min}$ ), the diabetics expelled stools but these were difficult to quantify because of their loose, watery composition. In general, control stools were always firm with a homogeneous white colour in contrast to the mottled appearance of diabetic stools. Diabetics continued to pass barium stools up to $48 \mathrm{~h}$ after the $1440 \mathrm{~min}$ radiograph whereas control animals rarely passed any barium stools after 630 minutes.

\section{Ultrastructure}

Fine structural analysis of the plexus of Auerbach indicated that autonomic neuropathology was confined to the distal, unmyelinated axons of diabetics. Excessive glycogen deposition was observed in certain axons of all diabetic nerve plexuses. Glycogen particles were heavily distributed throughout the axoplasm (Figs. 3, 4). In contrast, control axons were devoid of glycogen or possessed a few scattered particles (Fig. 5). Aggregates of neurofilaments were also seen in the axons of all diabetic plexuses. Filamentous elements were widespread in the axoplasm and interspersed among the glycogen particles (Fig. 3).
Neurofilaments were much less prominent in control axons (Fig. 5). Dense residual bodies were also scattered in the axoplasm of the pathologic fibres (Figs. 3, 4). Axons with excessive glycogen deposition and filamentous aggregation were more numerous in long-term ketonuric animals (5-9 months).

Hypertrophy or swelling of certain axons was evident in all diabetic plexuses (Figs. 3, 4). Swollen axons were characterized by an extensive increase in diameter (mean $4.2 \pm 0.3$ microns). In contrast to the uniform contour and diameter of normal axons (mean $0.6 \pm 0.2$ microns), the swollen fibres exhibited an irregular outline and width (Fig. 3). Glycogen deposition, filamentous accumulation and dense residual bodies were generally associated with the axoplasm of expanded axons (Figs. 3, 4).

Aggregates of lamellar inclusion bodies were seen in certain axons of $80 \%$ of the diabetic animals. These bodies were composed of numerous concentric membranes characteristic of residual lysosomes (Fig. 4). Dense core neurotransmitter vesicles were localized within some of the membranous whorls. Some vesicles within the membranes appeared to be in a degradative state (Fig. 4). The lamellar struc- 


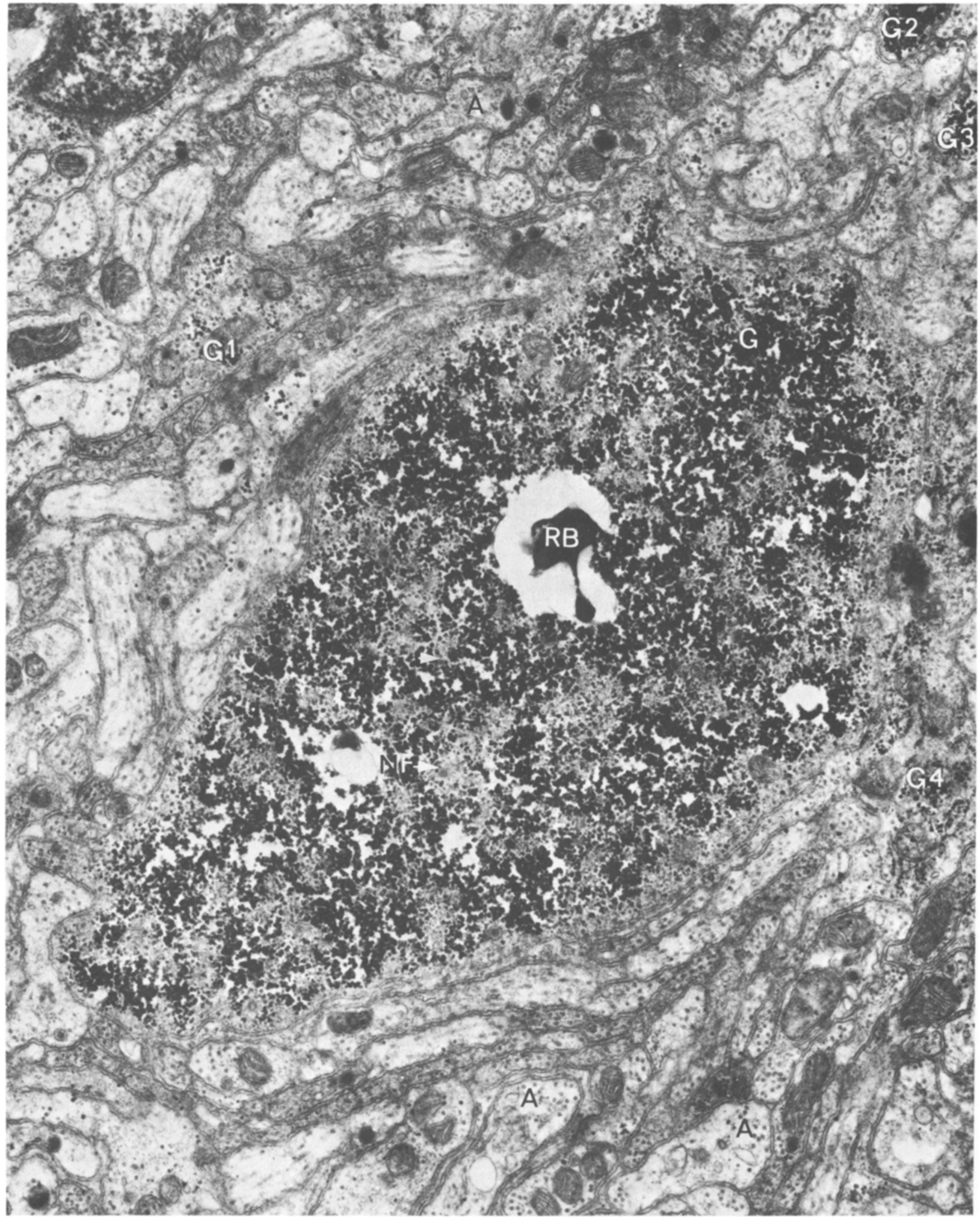

Fig. 3. Auerbach's plexus from Chinese hamster with 3 month duration of ketonuria. A swollen axon is visible in the center of the field with excess glycogen deposition $(\mathrm{G})$, fibrillar accumulation (NF) and a dense residual body (RB). Note the irregular contour of the expanded axon. In the field are normal appearing axons (A) as well as axons in the initial stages of glycogen accumulation (G1, G2, G3, G4). (approx. $21000 \times)$ 
tures were always located within swollen fibres and occupied almost the entire axoplasm. When lamellar bodies were aggregated in swollen axons, glycogen and neurofilaments were present in normal concentration. Lamellar inclusion bodies were found only in animals with long-term ketonuria (5-9 months).

\section{Discussion}

On barium radiology, the ketonuric diabetic Chinese hamster displayed significant impairment of gastric emptying, atony of the stomach and intestines, and reduction in intestinal peristaltic activity. Similar roentgenologic abnormalities are present in human diabetics $[4,5]$, but adequate control data are not available. The radiologic manifestation of gut dilatation in the Chinese hamster is consistent with previous morphometric studies which displayed significant enlargement of intestinal diameter $[16,17]$. Radiologic aberrations were observed in all diabetic hamsters. Radiologic abnormalities in human diabetics may be relatively uncommon unless the disease is of long duration and poorly controlled [7].

The overall barium pattern in the diabetic Chinese hamster implied that there was marked reduction in gastrointestinal motility. The effect of this complication was manifested as intestinal stasis. This has also been observed in diabetic man [4, 7]. This decrease in peristaltic activity is a conducive environment for intestinal bacterial overgrowth [19]. Significant elevated numbers of aerobic intestinal flora have been reported in the diabetic Chinese hamster [20]. Likewise, excessive bacterial multiplication has been seen in diabetic patients $[4,21,22]$. It is tempting to speculate that aerobic bacterial overgrowth is a major contributor to diarrhoea in the Chinese hamster. However, bacterial speciation and amelioration of diarrhoea by antibiotics will be necessary to substantiate this relationship. The effective therapeutic use of antibiotics in controlling some human cases of diabetic diarrhoea seems to support the concept that bacterial infection may be causally related to faecal incontinence [21, 23]. Unfortunately, the small number of patients and the lack of controls in these studies make it difficult to draw any unequivocal conclusions concerning the bacterial etiology of diabetic diarrhoea.

The data on the ultrastructural analysis of the Auerbach's plexuses showed that lesions were confined to the distal, unmyelinated axons. Excessive glycogen deposition, fibrillar accumulation and swelling were observed in certain axons of all diabetic animals. These aberrations affected a greater number of fibres per Auerbach's plexus as the duration of ketonuria increased. This implied that severity of neuropathology is linked to duration of ketonuria. It is uncertain whether excess glycogen in the axoplasm results from increased synthesis and storage or decreased utilization. Excessive glycogen deposition has previously been reported in retinal and kidney cells of ketotic Chinese hamsters [24] and in axons associated with several neurological disorders [25, 26]. The accumulation of fibrillar material in the axons of Auerbach's plexus may represent an initial stage of axonal degeneration in the diabetic Chinese hamster. This is the case in many other neuropathies [27]. Axonal fibrillar degeneration has also been reported in the distal, peripheral myelinated nerves of the diabetic Chinese hamster [28]. The pathogenesis of axonal swelling is highly speculative. Enlarged axons possessed aggregates of glycogen, fibrillar material or lamellar bodies. Thus, it is conceivable that overaccumulation of these inclusions contributed to the distention of axons.

The origin of lamellar body aggregates in the distal, naked axons is uncertain. However, on the basis of morphology, these inclusions seemed to resemble lysosomes which were in a secondary or residual state. They may have accumulated in response to excess glycogen deposition or may represent focal degradation of axons as observed in many other neuropathies [27]. This degradative phenomenon supports previous light microscopic studies which demonstrated necrotic axons in the myenteric plexuses of ketonuric Chinese hamsters [17]. The apparent remains of dense core transmitter vesicles were situated within certain lamellar bodies. This implicates a possible reduction in transmitter activity and agrees with earlier histochemical data which showed reduced cholinesterase activity at the intestinal neuromuscular junctions of diabetic Chinese hamsters [17]. It is also possible that lamellar bodies are the remnants of degenerate axoplasmic organelles or the end products of abnormal neuronal metabolism. The pathogenesis and significance of these hypotheses will have to be substantiated in future studies. Lamellar bodies were not found in animals of less than 5 months duration of ketonuria. This suggests that these inclusions may be a later complication of autonomic neuropathology in the Chinese hamster.

There are only scanty data on autonomic neuropathology in diabetic man. A few light microscopic studies have shown sporadic degeneration in the Auerbach's plexus, vagus and splanchnic nerves of diabetic patients $[10,14,15]$. These data are difficult to interpret due to variability, small sample size and the absence of controls. Indeed some investigators have reported an absence of lesions in the nervous innervation of the alimentary canal of diabetic patients [11]. In the ketonuric diabetic Chinese hamster, however, consistent pathologies have been observed in the distal, naked fibres of Auerbach's plexus. The autonomic fibres of Auerbach's plexus 


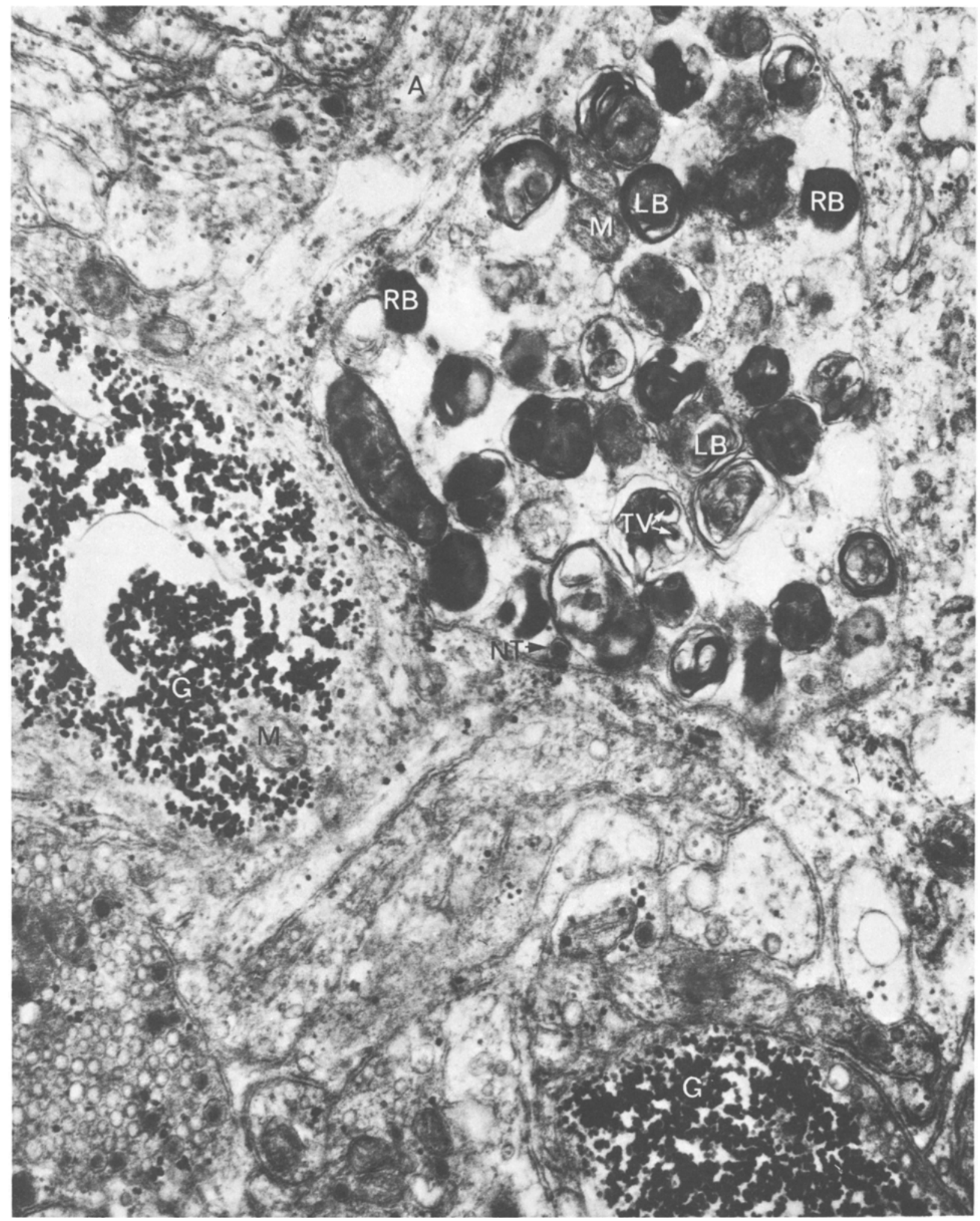

Fig. 4. Auerbach's plexus from Chinese hamster with 7 month duration of ketonuria. Note enlarged axons with glycogen deposition $(G)$, accumulation of lamellar bodies (LB) and dense residual bodies (RB). Two dense core neurotransmitter vesicles (TV) appear to be in a degenerative condition within a lamellar body. For comparison, a normal transmitter vesicle (NTV) is shown. Some normal mitochondria (M) are visible within the enlarged axons. Normal appearing axons (A) are found in this field. (approx. $32000 \times$ ) 


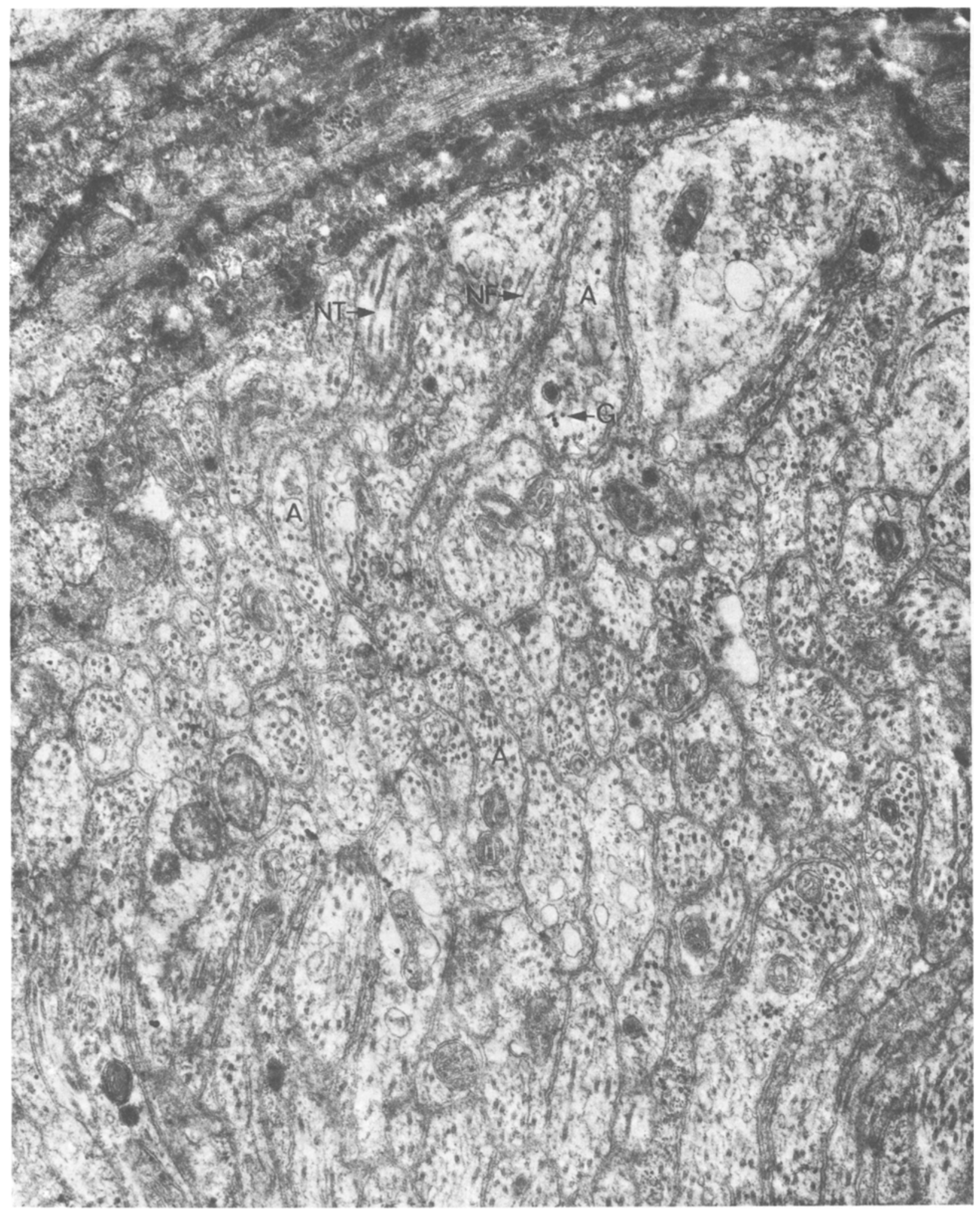

Fig. 5. Auerbach's plexus from control Chinese hamster. Note the normal size and appearance of naked axons (A) in this field. Glycogen particles $(\mathrm{G})$, neurotubules $(\mathrm{NT})$ and neurofilaments $(\mathrm{NF})$ are normally distributed. (approx. $21000 \times$ ) 
are responsible for innervation of intestinal musculature and control of peristaltic activity [29]. Any structural alterations in the myenteric plexuses would likely result in severe gastrointestinal dysfunction. Thus, it seems reasonable to conclude that autonomic neuropathology in the Auerbach's plexuses may be a primary factor underlying the atony, hypomotility, diarrhoea and bacterial overgrowth in the gastrointestinal tract of the ketonuric diabetic Chinese hamster.

Acknowledgements. The authors wish to thank the entire staff of the Hillcrest Hospital Radiology Department for technical assistance and advice during this investigation. Appreciation is also extended to Dr. W. E. Dulin for critical review of this manuscript. This work was supported in part by an Upjohn Company Grant and by Baylor University Summer Sabbatical (1977) and Baylor University Research Committee grants 002-76/7-BU-303 and 022-76/7-BU-303.

\section{References}

1. Mandelstam, P., Lieber, A.: Esophageal dysfunction in diabetic neuropathy-gastroenteropathy: clinical and roentgenological manifestations. JAMA 201, 88-92 (1967)

2. Wooten, R., Meriwether, J.: Diabetic gastric atony: a clinical study. JAMA 176, 68-73 (1961)

3. Howland, W., Drinkard, R.: Acute diabetic gastric atony: gastroparesis diabetacorum. JAMA 185, 144-146 (1963)

4. Katz, L., Spiro, H.: Gastrointestinal manifestations of diabetes. N. Engl. J. Med. 275, 1350-1361 (1966)

5. Zitomer, B., Gramm, H., Kozak, G.: Gastric neuropathy in diabetes mellitus: clinical and radiologic observations. Metabolism 17, 199-211 (1968)

6. Hodges, F., Rundles, W., Hanelin, J.: Roentgenologic study of the small intestine. II. Dysfunction associated with neurologic diseases. Radiology 49, 659-673 (1947)

7. McNally, E., Reinhard, A., Schwartz, P.: Small bowel motility in diabetics. Am. J. Dig. Dis. 14, 163-169 (1969)

8. Berge, K., Wollaeger, E., Scholz, D., Rooke, E., Sprague, R.: Steatorrhea complicating diabetes mellitus with neuropathy: report of cases without apparent external pancreatic insufficiency. Diabetes 5, 25-31 (1956)

9. Ellenberg, M., Bookman, J.: Diabetic diarrhea with malabsorption syndrome. Diabetes 9, 14-18 (1960)

10. Rundles, W.: Diabetic neuropathy: general review with report of 125 cases. Medicine (Baltimore) 24, 111-160 (1945)

11. Berge, K., Sprague, R., Bennett, W.: The intestinal tract in diabetic diarrhea: a pathologic study. Diabetes 5, 289-294 (1956)

12. Appenzeller, O., Richardson, E.: The sympathetic chain in patients with diabetic and alcoholic polyneuropathy. Neurology (Minneap.) 16, 1205-1209 (1966)

13. Hensley, G., Soergel, K.: Neuropathologic findings in diabetic diarrhea. Arch. Pathol. Lab. Med. 85, 587-597 (1968)
14. Kristensson, K., Nordborg, C., Olsson, Y., Sourander, P.: Changes in the vagus nerve in diabetes mellitus. Acta Pathol. Microbiol. Scand. 79, 684-685 (1971)

15. Low, P., Walsh, J., Huang, C., McLeod, J.: The sympathetic nervous system in diabetic neuropathy. Brain 98, 341-356 (1975)

16. Diani, A., Gerritsen, G., Stromsta, S., Murray, P.: A study of the morphological changes in the small intestine of the spontaneously diabetic Chinese hamster. Diabetologia 12, 101-109 (1976)

17. Bryan, F., Diani, A., Gerritsen, G.: A comparative study of histopathology in the small intestine of nonketotic diabetic and ketotic diabetic Chinese hamsters. Diabetes 26, 144 (1977) (abstract)

18. Gerritsen, G., Blanks, M.: Characterization of Chinese hamsters by metabolic balance, glucose tolerance and insulin secretion. Diabetologia 10, 493-499 (1974)

19. Gorbach, S.: Studies of intestinal microflora: effects of diet, age and periodic sampling on numbers of fecal microorganisms in man. Gastroenterology 53, 845-855 (1967)

20. Eldridge, D., Yates, M., Stutsman, S., Risinger, D., Diani, A.: Microbiologic and radiologic abnormalities in the gastrointestinal tract of the ketotic diabetic hamster. Diabetes 27, 475 (1978) (abstract)

21. Goldstein, F., Wirts, C., Kowlessar, O.: Diabetic diarrhea and steatorrhea, microbiologic and clinical observations. Arch. Intern. Med. 72, 315-318 (1977)

22. Whalen, G., Soergel, K., Green, J.: Diabetic diarrhea: a clinical and pathophysiological study. Gastroenterology 56, 1-21-1032 (1969)

23. Malins, J., French, J.: Diabetic diarrhea. Q. J. Med. 26, $467-480$ (1957)

24. Soret, M., Dulin, W., Mathews, J., Gerritsen, G.: Morphologic abnormalities observed in retina, pancreas and kidney of diabetic Chinese hamsters. Diabetologia 10, 567-570 (1974)

25. Dyck, P., Thomas, P., Lambert, E.: Peripheral neuropathy, Volume 2, p. 1003-1006. Philadelphia, London, Toronto: Saunders 1975

26. Powell, H., Knox, D., Lee, S.: Alloxan diabetic neuropathy: electron microscopic studies. Neurology (Minneap.) 27, 60-66 (1977)

27. Dyck, P., Thomas, P., Lambert, E.: Peripheral neuropathy, Volume 1, p. 325. Philadelphia, London, Toronto: Saunders 1975

28. Schlaepfer, W.: Segmental demyelination in the distal peripheral nerves of chronically diabetic Chinese hamsters. Diabetologia 10, 541-548 (1974)

29. Noback, D., Demarest, R.: The human nervous system: Basic principles of neurobiology, p. 205. New York, St. Louis, San Francisco: McGraw Hill 1975

Received: August 2, 1978, and in revised form: February 1, 1979

A. R. Diani, Ph. D.

Department of Anatomy

East Carolina University School of Medicine

Greenville, NC 27834

USA 